\title{
Are specialized pro-resolving mediators promising therapeutic agents for severe bronchial asthma?
}

\author{
Takeshi Hisada, Haruka Aoki-Saito, Yasuhiko Koga \\ Department of Respiratory Medicine, Gunma University Graduate School of Medicine, Maebashi, Gunma, Japan \\ Correspondence to: Takeshi Hisada, MD, PhD. Department of Respiratory Medicine, Gunma University Graduate School of Medicine, 3-39-15, \\ Showa-machi, Maebashi, Gunma 371-8511, Japan. Email: hisadat@gunma-u.ac.jp.
}

Submitted Sep 23, 2017. Accepted for publication Oct 10, 2017.

doi: $10.21037 /$ jtd.2017.10.116

View this article at: http://dx.doi.org/10.21037/jtd.2017.10.116

In Japan, although the number of patients with asthma has increased, the number of patients who die from asthma has decreased (1.2 per 100,000 patients in 2015) (1). Currently, although the majority of asthma patients can be effectively treated with available medications, such as inhaled corticosteroids (ICS) or ICS/long-acting $\beta_{2}$ agonists (ICS/ LABA), options remain to be established for difficult-totreat asthma (severe asthma), which can be considered an unmet need. Patients with severe asthma (presumably less than $10 \%$ of all asthma cases) who experience exacerbations consume considerable healthcare resources (2). According to data from a U.S. study by Chastek et al., in 2013, the total adjusted asthma-related costs among patients with severe asthma were $\$ 5,112$ per year, or 2.9-fold higher than that among patients with persistent asthma (3). Adjusted asthma medication costs were $\$ 4,020$ per year, which was triple the asthma medication costs for patients with persistent asthma. Much remains unclear regarding the optimal approach for the management of these patients and the underlying mechanisms driving this disease. Asthma is characterized by airway hyperresponsiveness and chronic airway inflammation. Numerous inflammatory cells, including eosinophils and lymphocytes, infiltrate the peribronchial tissue in patients with asthma. Long-standing asthma induces airway remodeling, leading to intractable asthma. Asthma patients are characterized by widely variable clinical pictures; asthma is thus often recognized as a syndrome and is classified into several phenotypes. Recent progress in genetics and molecular biology has led to the etiological and/or pathogenetic classification of the condition into endotypes (4). Recent studies have uncovered the actions and clinical potential of specialized pro-resolving mediators (SPMs), namely, the superfamily of pro-resolving mediators that includes the resolvin (E-series, D-series, and DPA-derived), protectin, and maresin families, as well as arachidonic-acid-derived lipoxins (5). Resolvin E1 (RvE1) is an anti-inflammatory lipid mediator derived from the omega-3 fatty acid, eicosapentaenoic acid (EPA), and has been recently shown to be involved in resolving inflammation (6). Although little is known about the actions of RvE1 in the resolution of asthma-induced inflammation, recent studies using a mouse model have shown the potential of RvE1 in treating asthma (7-9). In this editorial, we assess SPMs as possible candidates for asthma (particularly severe asthma) treatment, and review the current understanding of their mechanism of action in connection with asthma pathogenesis and treatment. Studies have provided evidence for the use of RvE1 and other SPMs as a pivotal counter-regulatory signal in allergic inflammation, offering the possibility of novel therapeutic approaches for human asthma and also severe asthma, particularly in certain asthma endotypes. SPMs and their analogues might be useful for the treatment of asthma.

\section{Effect of SPMs in bronchial asthma}

Resolution of inflammation is characterized by clearance of inflammatory leukocytes from the lung, restoration of epithelial barrier function, and dampening of airway hyperreactivity. During resolution, multiple homeostatic mechanisms are enlisted to generate endogenous "braking signals". Several classes of counter-regulatory lipid mediators have been recently discovered that are generated from polyunsaturated fatty acids (PUFAs) during inflammation to promote resolution (10). 


\section{Protectin D1 (PD1)}

Protectins are identified as natural chemical mediators that counter leukocyte activation to promote resolution of inflammation. Levy et al. reported the first evidence that PD1 was formed from docosahexaenoic acid (DHA) in human asthma and had counter-regulatory actions in allergic airway inflammation (11). PD1 was present in exhaled breath condensates (EBC) from healthy subjects. The levels of PD1 were lower in the EBC of asthmatic patients than in that of healthy subjects. In experiments with mice, PD1 was administrated intravenously before or after aeroallergen challenge, and the number of eosinophil and lymphocyte cells decreased, as did the levels of interleukin (IL)-13, prostaglandin $\mathrm{D}_{2}\left(\mathrm{PGD}_{2}\right)$, and cysteinyl leukotrienes (CysLTs) in bronchoalveolar lavage fluid (BALF) and airway hyperresponsiveness to inhaled methacholine. Interestingly, PD1 did not change the level of IL-5 in BALF. These results suggest protective effects on asthmatic responses in vivo without changes of IL-5 concentration in BALF. Toll-like receptor 7 (TLR7) is necessary for the recognition of the single-stranded RNA of respiratory viruses. TLR7 agonists exert preventive effects on allergic airway inflammation in vivo in mice and function as bronchodilators in humans, indicating that they are potential therapeutic targets in asthma. Interestingly, TLR7 signaling promotes the resolution of airway eosinophilic inflammation through upregulation of 12/15-lipoxygenase metabolism, and its metabolites, such as PD1 and resolvin D1 (RvD1), also demonstrate suppressive effects (12). The receptor for PD1 remains unknown, although its existence in neutrophils, pigment epithelial cells, and neuronal cells has been suggested.

\section{RvD1, aspirin-triggered RvD1 (AT-RvD1), and RvD2}

Both RvD1 and AT-RvD1 administered intravenously inhibited airway eosinophil accumulation and mucus production with decreased IL-5 and IL-17 production in a murine model of asthma. Aspirin-triggered RvD1 considerably reduced inflammatory cells (eosinophils and lymphocytes), lipid mediators, and airway hyperresponsiveness relative to RvD1 (13). RvD1 and ATRvD1 promoted alveolar macrophage phagocytosis in vitro, suggesting that they enhance the clearance of apoptotic inflammatory cells in the airway. Hsiao et al. reported that RvD1 and AT-RvD1 accelerated the resolution of lung inflammation, driving macrophage polarization from phenotype M1 to M2 (14). Moreover, Martin et al. reported that RvD1 and RvD2 attenuated the release of histamine from human lung mast cells (15). These reports could also indicate the anti-asthmatic mechanisms of RvD1 and RvD2. ALX/FPR2 and GPR32 were identified as high-affinity receptors for RvD1.

\section{RvE1}

Numerous reports have demonstrated the protective effects of RvE1 on airway eosinophilic inflammation in vivo and in vitro (7-9). First, we reported that RvE1 dampens airway inflammation and airway hyperresponsiveness in a murine asthma model. Treatment with intraperitoneal administration of RvE1 during the sensitization phase, challenge phase, or both inhibits OVA-specific IgE in serum, inflammatory cell accumulation in the airways, airway hyperresponsiveness to methacholine, mucus production, and Th2 cytokine (IL-13, IL-5) production in BALF. Miyahara et al. reported that leukotriene $\mathrm{B}_{4}\left(\mathrm{LTB}_{4}\right)$ and block lipid transport-1 (BLT1) play crucial roles in Th2 cytokine IL-13 production by lung T cells and in the recruitment of antigen-specific effector $\mathrm{CD} 8^{+} \mathrm{T}$ cells (16). The $\mathrm{LTB}_{4}$-BLT1 pathway is a critical target for the treatment of bronchial asthma. We suggest that RvE1 acts partly as a potent antagonist for BLT1. On the other hand, Haworth et al. demonstrated that $\mathrm{RvE} 1$ regulates IL-23, interferon- $\gamma$ (IFN- $\gamma$ ), and lipoxin $\mathrm{A}_{4}$ to promote the resolution of allergic airway inflammation in a mouse model of asthma. RvE1 administered intravenously during the resolution phase also dampened inflammatory cell accumulation in the airways, airway hyperresponsiveness, and mucus production. RvE1 effected that the inhibition of IL-17A, IL-23, IL-6, IL-27, LTB 4 , T-helper 17, and $\mathrm{LTB}_{4}$, but not IL-10, IL-4, IL-5, IL-13, PGD 2 , or CysLTs in BALF and serum immunoglobulin E. RvE1binds to two receptors, namely, chemokine-like receptor 1 (ChemR23) and $\mathrm{LTB}_{4}$ receptor BLT1.In humans and mice, ChemR23 is expressed on monocytes, macrophages, dendritic cells (DCs), natural killer (NK) cells, platelets, vascular smooth muscle cells, and endothelial cells. However, the expression of ChemR23 in $\mathrm{CD}^{+} \mathrm{T}$ lymphocytes is lower than that of other inflammatory cells. Haworth et al. suggested that RvE1 directly modulates cytokine production by dendritic cells and activates NK cells, the main producers of IFN- $\gamma$ and active inducers of eosinophil apoptosis (17). In addition, it was also reported that RvE1-exposed DCs attenuated the chemotaxis of splenic DCs and bone marrow DCs to the 
lymph nodes. These results suggest $\mathrm{RvE} 1$ may play a role in various aspects of the resolution phase.

Regarding strategies for severe asthma, bronchial thermoplasty (BT) has recently been proposed as a novel intervention that delivers controlled thermal energy to the airway wall during a series of bronchoscopy procedures, resulting in a prolonged reduction in airway smooth muscle mass. BT therapy has been approved in Japan since 2015 . Only one report has stated that BT in patients with severe persistent asthma improves asthma-specific quality of life, with a reduction in severe exacerbations and healthcare use at 5 years post-treatment (18). However, the longterm efficacy and safety of this therapy remain unknown and should be studied in future. Other new therapeutic strategies have also been revealed. Antibodies against IL-5 and IL-13 have been developed for severely asthmatic patients. The anti-IL-5 antibody, mepolizumab, reduces asthmatic exacerbations, improves respiratory symptoms and pulmonary function, and can reduce the required doses of oral steroids in patients with higher blood eosinophil counts. Mepolizumab therapy has been approved in Japan since 2016. The anti-IL-13 antibody and anti-IL-4 receptor -chain antibody improve pulmonary function in moderately to severely asthmatic patients and also reduces the frequency of exacerbations with a reduction of ICS. An anti-thymic stromal lymphopoietin antibody and anti-IL-17 antibody are still under development. In a review study in 2008, Anderson found strong evidence suggesting that resolution processes are defective in asthma and this could be one endotype of severe asthma (4). Miyata et al. determined thatPD1 is synthesized in human eosinophils (19). At nanomolar concentrations, PD1suppressed the chemotaxis induced by $\mathrm{C}-\mathrm{C}$ motif chemokine ligand 11/eotaxin-1 or 5 -oxo-eicosatetraenoic acid and modulated the expression of the adhesion molecules CD11b and L-selectin, although it exerted no effects on the degranulation, superoxide anion generation, or survival of eosinophils. Compared to that in cells harvested from healthy subjects, they observed a notable decrease in the biosynthesis of PD1 by eosinophils in patients with severe asthma, even in the presence of DHA. These observations are the first indication that activated human eosinophils represent a major source of PD1, which can act as self-resolving machinery in eosinophilic inflammation, whereas the production of PD1 is impaired in patients with severe asthma.

Thus, the question is raised as to whether abnormalities in the production or function of $\mathrm{RvE} 1, \mathrm{RvE} 3$ and PD1 are causes of intractable asthma. To challenge intractable asthma in relation to resolvins and other SPMs, we must investigate these fields further. SPMs are unstable in oxygen because of their characteristic polyunsaturated structures, and numerous problems exist in their clinical application. Possible solutions to these problems are provided in a study by Fukuda et al. (20), in whichCP-RvE2 (the cisolefin of RvE2 being replaced with a cyclopropane) was designed and synthesized. The novel CP-RvE2 was much more stable than $\mathrm{RvE} 2$ against autoxidation and equipotent to or more potent than RvE2, and was identified as a successful, stable equivalent of RvE2. As a stable equivalent, lipid chemical mediator resolvins with highly potent antiinflammatory activity can lead the way in the development of novel anti-inflammatory drugs. Asthma is a heterogenous syndrome of chronic airway inflammation and has many endotypes. At least for the SPM-deficient endotype or non-functional subtype, we believe that SPMs and/or their stable equivalents will be promising strategic agents as new therapeutic options for severe asthma.

\section{Acknowledgements}

None.

\section{Footnote}

Conflicts of Interest: The authors have no conflicts of interest to declare.

\section{References}

1. Ichinose $M$, Sugiura H, Nagase Het al. Japanese guidelines for adult asthma 2017. Allergol Int 2017;66:163-89.

2. Chung KF, Wenzel SE, Brozek JL, et al. International ERS/ATS guidelines on definition, evaluation and treatment of severe asthma. Eur Respir J 2014;43:343-73.

3. Chastek B, Korrer S, Nagar SP, et al. Economic Burden of Illness Among Patients with Severe Asthma in a Managed Care Setting. J Manag Care Spec Pharm 2016;22:848-61.

4. Anderson GP. Endo typing asthma: new insights into key pathogenic mechanisms in a complex, heterogeneous disease. Lancet 2008;372:1107-19.

5. Serhan CN. Discovery of specialized pro-resolving mediators marks the dawn of resolution physiology and pharmacology. Mol Aspects Med 2017;58:1-11.

6. Hisada $\mathrm{T}$, Ishizuka $\mathrm{T}$, Aoki $\mathrm{H}$, et al. ResolvinE1 as a novel agent for the treatment of asthma. Expert Opin Ther Targets 2009;13:513-22. 
7. Aoki H, Hisada T, Ishizuka T, et al. Resolvin E1 dampens airway inflammation and hyperresponsiveness in a murine model of asthma. Biochem Biophys Res Commun 2008;367:509-15.

8. Haworth O, Cernadas M, Yang R, et al. Resolvin E1 regulates interleukin 23, interferon- $\gamma$ and lipoxin $\mathrm{A} 4$ to promote the resolution of allergic airway inflammation. Nat Immunol 2008;9:873-9.

9. Aoki H, Hisada T, Ishizuka T. Protective effect of resolvin E1 on the development of asthmatic airway inflammation. Biochem Biophys Res Commun 2010;400:128-33.

10. Serhan CN, Chiang N, Van Dyke TE. Resolving inflammation: dual anti-inflammatory and pro-resolution lipid mediators. Nat Rev Immunol 2008;8:349-61.

11. Levy BD, Kohli P, Gotlinger K, et al. Protectin D1 is generated in asthma and dampens airway inflammation and hyperresponsiveness. J Immunol2007;178:496-502.

12. Koltsida O, Karamnov S, Pyrillou K, et al. Toll-like receptor 7 stimulates production of specialized proresolving lipid mediators and promotes resolution of airway inflammation. EMBO Mol Med 2013;5:762-75.

13. Rogerio AP, Haworth O, Croze R, et al. Resolvin D1 and aspirin-triggered resolvin D1 promote resolution of allergic airways responses. J Immunol 2012;189:1983-91.

14. Hsiao HM, Sapinoro RE, Thatcher TH, et al. A novel

Cite this article as: Hisada T, Aoki-Saito H, Koga Y. Are specialized pro-resolving mediators promising therapeutic agents for severe bronchial asthma? J Thorac Dis 2017;9(11):4266-4269. doi: 10.21037/jtd.2017.10.116 anti-inflammatory and pro-resolving role for resolvin D1 in acute cigarette smoke-induced lung inflammation. PLoS One 2013;8:e58258.

15. Martin N, Ruddick A, Arthur GK, et al. Primary human airway epithelial cell-dependent inhibition of human lung mast cell degranulation. PLoS One2012;7:e43545.

16. Miyahara N, Takeda K, Miyahara S, et al. Leukotriene B4 receptor- 1 is essential for allergen-mediated recruitment of CD8+ T cells and airway hyperresponsiveness. J Immunol 2005;174:4979-84.

17. Haworth O, Cernadas M, Levy BD. NK cells are effectors for resolvin $\mathrm{E} 1$ in the timely resolution of allergic airway inflammation. J Immunol 2011;186:6129-35.

18. Wechsler ME, Laviolette M, Rubin AS, et al. Bronchialthermoplasty: Long-term safety and effectiveness in patients with severe persistent asthma. J Allergy Clin Immunol 2013;132:1295-302.

19. Miyata J, Fukunaga K, Iwamoto R, et al. Dysregulated synthesis of protectin D1 in eosinophils from patients with severe asthma. J Allergy Clin Immunol 2013;131: 353-60.e1-2.

20. Fukuda H, Muromoto R, Takakura Y, et al. Design and Synthesis of Cyclopropane Congeners of ResolvinE2, an Endogenous Proresolving Lipid Mediator, as Its Stable Equivalents. Org Lett 2016;18:6224-7. 